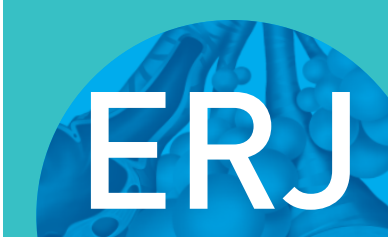

open research
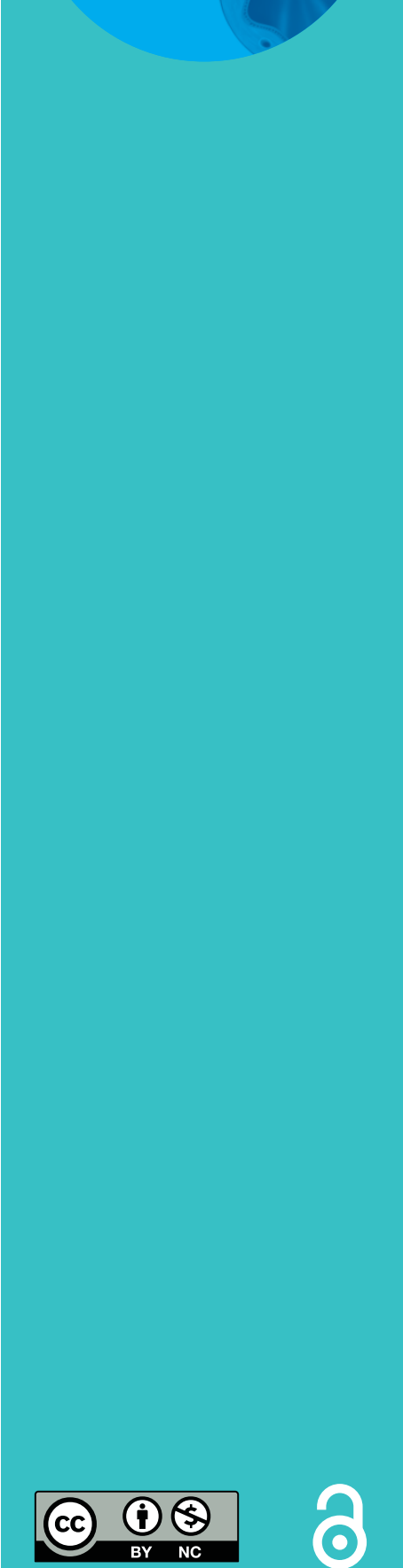

\section{Amikacin exposure and susceptibility of macrolide-resistant Mycobacterium} abscessus

\author{
Shelby Daniel-Wayman ${ }^{1}$, Shamira Shallom², Nabila Azeem², \\ Kenneth N. Olivier ${ }^{3}$, Adrian M. Zelazny ${ }^{2}$ and D. Rebecca Prevots ${ }^{1}$
}

Affiliations: ${ }^{1}$ Epidemiology Unit, Laboratory of Clinical Immunology and Microbiology, National Institute of Allergy and Infectious Diseases, National Institutes of Health, Bethesda, MD, USA. ${ }^{2}$ Dept of Laboratory Medicine, Clinical Center, National Institutes of Health, Bethesda, MD, USA. ${ }^{3}$ Laboratory of Chronic Airway Infection, Pulmonary Branch, National Heart, Lung, and Blood Institute, National Institutes of Health, Bethesda, MD, USA.

Correspondence: D. Rebecca Prevots, Epidemiology Unit, Laboratory of Clinical Immunology and Microbiology, National Institute of Allergy and Infectious Diseases, National Institutes of Health, 8 West Drive, MSC 2665, Bethesda, MD 20892, USA. E-mail: rprevotsanih.gov

ABSTRACT Mycobacterium abscessus is associated with antibiotic resistance and poor treatment outcomes. We described within-patient changes in M. abscessus resistance to clarithromycin and amikacin.

Patients with amikacin exposure and a >50-month interval between $M$. abscessus isolates were identified. Antimicrobial susceptibility testing was performed on the first and last isolates by broth microdilution, and genetic markers of resistance were identified.

16 patients were identified with a median amikacin exposure of 2.3 years (range 0.6-8.6 years). 15 patients also received macrolides (median 7.2 years, range $1.3-10.7$ years). All initial isolates were resistant to clarithromycin (minimum inhibitory concentration (MIC) $\geqslant 8 \mu \mathrm{g} \cdot \mathrm{mL}^{-1}$ ). Two patients had later susceptible isolates, which were of a different subspecies (M. abscessus subsp. massiliense) than the initial isolates (M. abscessus subsp. abscessus). All initial isolates were susceptible or intermediately resistant to amikacin, and only one patient had a resistant final isolate (MIC $>64 \mu \mathrm{g} \cdot \mathrm{mL}^{-1}$ ), accompanied by an $\mathrm{A} \rightarrow \mathrm{G}$ mutation at position 1408 of the $16 \mathrm{~S}$ ribosomal RNA. Forced expiratory volume in $1 \mathrm{~s}$ decreased significantly over the study period, while smear quantity and the proportions of patients with elevated C-reactive protein or cavitary lesions all increased significantly.

Despite prolonged, mostly inhaled amikacin exposure, development of amikacin resistance was uncommon in this patient population; however, disease progression continued.

@ERSpublications

Patients with long-term amikacin treatment rarely develop resistance but their disease continues to progress http://bit.ly/2 $\mathrm{V} 7 \mathrm{k} 0 \mathrm{kH}$

Cite this article as: Daniel-Wayman S, Shallom S, Azeem N, et al. Amikacin exposure and susceptibility of macrolide-resistant Mycobacterium abscessus. ERJ Open Res 2019; 5: 00154-2018 [https://doi.org/10.1183/23120541.00154-2018].

This article has supplementary material available from openres.ersjournals.com

Received: Sept 072018 | Accepted after revision: April 152019

The content of this work is not subject to copyright. Design and branding are copyright @ERS 2019. This article is open access and distributed under the terms of the Creative Commons Attribution Non-Commercial Licence 4.0. 


\section{Introduction}

Nontuberculous mycobacteria (NTM) are environmental mycobacteria associated with chronic lung disease, with increasing prevalence in the USA, Europe and Asia [1,2]. Mycobacterium abscessus is the second most common NTM species [3], with poor treatment outcomes and resistance to most antibiotics $[4,5]$. M. abscessus is comprised of three closely related sub-taxa, which will be referred to in this paper as M. abscessus subsp. abscessus, M. abscessus subsp. massiliense and M. abscessus subsp. bolletii [6].

The 2007 American Thoracic Society NTM guidelines note that for M. abscessus, no drug regimen has been shown to produce long-term sputum conversion, but suggest a macrolide (clarithromycin or azithromycin) with amikacin plus cefoxitin or imipenem as noncurative therapy [7]. Cure rates may depend upon macrolide resistance, with one study finding sputum conversion in $88 \%$ of patients with primarily clarithromycin-susceptible $M$. abscessus subsp. massiliense, compared with $25 \%$ in patients with M. abscessus subsp. abscessus, which is commonly clarithromycin resistant [8]. Amikacin has been associated with clinical and microbiological improvement in both intravenous [8] and inhaled [9] forms.

M. abscessus exhibits both acquired and inducible forms of macrolide resistance. Acquired resistance is conferred by mutations at position 2058 (A2058G/C/T) or 2059 (A2059G/C) in the 23S ribosomal RNA (rRNA) $r r l$ gene $[10,11]$. Inducible resistance, expressed following macrolide exposure in $M$. abscessus subsp. abscessus, requires a full length and functional erm(41) gene [12-15]. In contrast, most M. abscessus subsp. massiliense strains lack inducible resistance due to a 274-bp deletion in the erm(41) gene that renders it nonfunctional $[8,14]$. An intact erm(41) gene can also be rendered nonfunctional by a $\mathrm{T} \rightarrow \mathrm{C}$ mutation at position 28 [13]. Amikacin resistance is conferred by single base pair mutations in the $16 \mathrm{~S}$ rRNA gene (rrs): T1406A, A1408G, C1409T and G1491T [16, 17].

Relatively little research has focused on the development of amikacin resistance within M. abscessus and its relevance to clinical management. One retrospective study of amikacin treatment in refractory Mycobacterium avium complex (MAC) and M. abscessus patients found a $6 \%$ prevalence of amikacin resistance initially, with no resistance development detected in $M$. abscessus patients in follow-up testing within 12 months [18]. In a trial of liposomal amikacin for inhalation (LAI), nine out of 89 (10\%) MAC and M. abscessus patients enrolled in the study had high-level (minimum inhibitory concentration (MIC) $\left.>64 \mu \mathrm{g} \cdot \mathrm{mL}^{-1}\right)$, mutation-confirmed amikacin resistance at the start and five patients developed resistance during the study [19]. In both studies, none of the patients with amikacin resistance achieved culture conversion during the study period [18, 19]. However, most patients had MAC infections, and the development of amikacin resistance while on treatment has not been studied for M. abscessus alone or after long treatment durations. We conducted a pilot study to identify changes in amikacin resistance among patients with serial isolates who have undergone prolonged antibiotic treatment. Additionally, we identified genetic markers of resistance and assessed changes in clinical, radiographic and microbiological measures over the study period.

\section{Materials and methods}

Records from patients enrolled in institutional review board-approved natural history studies (www. clinicaltrials.gov identifier numbers NCT00018044 or NCT00943514) [20], for which patients provided informed consent, were queried for the period January 1, 2005 to November 28, 2016 to identify patients with $M$. abscessus isolates. For the purposes of the pilot study, we selected the 16 patients with the longest follow-up ( $>50$ months) between sequential isolates who received $\geqslant 3$ months of treatment with amikacin sulfate as part of a multidrug regimen. Most patients received inhaled amikacin with dosing as previously described ranging from 250-500 mg once or twice daily [9]. Through selection of patients with the longest duration of amikacin exposure, we maximised the likelihood of detecting development of amikacin resistance. Data were abstracted from medical records for the visits closest in time to the initial and final cultures, with visits $>365$ days from sample collection excluded. C-reactive protein (CRP) was dichotomised at the upper limit of normal and severity of bronchiectasis was dichotomised as a Reiff score $>3$ [21]. Culture conversion was defined as three consecutive sputum cultures negative for M. abscessus, collected $\geqslant 4$ weeks apart [22]. For analysis of bacterial load, the highest culture quantitation at each timepoint was used [9]. Statistical significance $(p<0.05)$ of differences between timepoints was assessed with a paired t-test, Wilcoxon-signed rank test or McNemar's test.

\section{Susceptibility testing}

Antimicrobial susceptibility testing was performed by broth microdilution in Mueller-Hinton medium, using Sensititre RAPMYCO plates (Trek Diagnostic Systems; Thermo Fisher Scientific, Oakwood Village, $\mathrm{OH}$, USA) according to Clinical and Laboratory Standards Institute (CLSI) M24-A2 guidelines [23]. Plates were evaluated at 3-5 days; clarithromycin MICs were additionally read at 14 days to detect inducible 
resistance. Consecutive isolates showing changes in amikacin resistance underwent repeat testing for confirmation.

\section{Sequencing of 165 rRNA, 23 S rRNA and erm(41) genes}

Strains were stored at $-80^{\circ} \mathrm{C}$ in Tween albumin broth (Remel, Lenexa, KS, USA) and subcultured onto Middlebrook 7H11 agar (Remel). DNA was extracted with the Ultra Clean microbial DNA isolation kit (MoBio Laboratories, Solana Beach, CA, USA). Partial amplification and sequencing of 16S rRNA (rrs1-F and rrs1-R [16]), 23S rRNA (23S_18F, 23S_21R, 23SrRNAF_207 and 23SrRNAR_207R [24]) and erm(41) (ermF and ermR1 [14]) genes was performed on all study isolates, and compared to M. abscessus subsp. abscessus ATCC $19977^{\mathrm{T}}$, M. abscessus subsp. massiliense CCUG $48898^{\mathrm{T}}$ and M. abscessus subsp. bolletii $\mathrm{BD}^{\mathrm{T}}$ (CIP108541) (primers from IDT, Coralville, IA, USA). For all PCR reactions, $250 \mathrm{ng}$ of genomic DNA was amplified and sequenced as described previously [25]. SeqMan Pro (version 13.0.2; DNAStar, Inc., Madison, WI, USA) was used for sequence assembly. Multiple-sequence alignment was carried out using the CLUSTALW algorithm in MegAlign (version 13.0; DNAStar, Inc.). Sequencing of $\sec A, r p o B$ and hsp65 genes [26] was also conducted on all samples to help confirm that the first and last isolates for each patient were the same strain.

\section{Results}

\section{Demographics and antibiotic exposure}

99 patients were identified with $M$. abscessus. 63 patients had multiple sequential isolates available for testing, of whom 34 had $>3$ months of cumulative exposure to amikacin. 16 patients with the greatest duration between isolates were selected, and 32 initial and final positive sputum cultures identified. For six cultures, multiple distinct mycobacterial colony types were noted, indicating significant heterogeneity of infecting strains. Isolation of different colony morphologies for identification is CLSI-recommended practice. Thus, for these cultures, an example of each colony type was isolated and identified, for a total of 38 M. abscessus isolates. The average interval between samples was 7.6 years (range 4.3-10.7 years) (table 1). Of the 16 patients, two had cystic fibrosis, one had primary ciliary dyskinesia and 13 had idiopathic bronchiectasis (table 1).

During the study period, patients were treated with amikacin for a median of 2.5 years (range 0.6-8.6 years) (tables 2 and 3, and figure 1). All patients received inhaled amikacin therapy during the study period, while only three patients received intravenous amikacin. Prior to the study period, eight out of 16 patients received treatment with either inhaled amikacin, intravenous amikacin or both. Of these, five patients were treated with intravenous amikacin, including two of the three patients who received it during the study period. Intravenous amikacin use was often limited by toxicity. Macrolides were also prescribed to 15 of the 16 patients during the study period, with a median duration of 7.2 years (range 1.3-10.7 years) (tables 2 and 3). Patients were additionally treated for M. abscessus with combinations of other medications (table 3 ).

Antibiotic susceptibility test results, and sequencing of 16S rRNA, 23S rRNA and erm(41) genes Most patients (15 out of 16 ) had both first and last isolates that tested susceptible (MIC $<16 \mu \mathrm{g} \cdot \mathrm{mL}^{-1}$ ) or intermediately resistant (MIC $32 \mu \mathrm{g} \cdot \mathrm{mL}^{-1}$ ) to amikacin. Only one patient (K) had a last isolate (K2) resistant (MIC $>64 \mu \mathrm{g} \cdot \mathrm{mL}^{-1}$ ) to amikacin; this patient's first isolate was susceptible (MIC $16 \mu \mathrm{g} \cdot \mathrm{mL}^{-1}$ ) (table 4). Isolate $\mathrm{K} 2$ had an A1408G mutation of the $16 \mathrm{~S}$ rRNA (table S1) known to confer aminoglycoside resistance in M. abscessus [16].

In the time between isolates $\mathrm{K} 1$ and $\mathrm{K} 2$, this patient received 611 days of amikacin treatment, including 30 days of intravenous amikacin. However, medical records indicate that this patient had a prior resistant isolate grown from a sample taken 324 days after the initial susceptible isolate (K1) (figure 1). During these first 324 days, the patient had 241 days of inhaled amikacin treatment.

\section{TABLE 1 Baseline patient characteristics}

Patients

Age at initial sample years median (range) 
TABLE 2 Exposure to selected antibiotics over the study period

\begin{tabular}{lcc} 
Antibiotic & Patients & Time for which used years median (range) \\
\hline Amikacin\# & $16(100 \%)$ & $2.3(0.56-8.6)$ \\
Inhaled amikacin including LAl & $16(100 \%)$ & $2.3(0.56-7.7)$ \\
Intravenous amikacin & $3(19 \%)$ & $0.16(0.08-0.88)$ \\
Macrolide & $15(94 \%)$ & $7.2(1.3-10.7)$ \\
\end{tabular}

LAl: liposomal amikacin for inhalation. ${ }^{\#}$ : includes LAl in the context of a clinical trial for six patients. Of these, four patients participated in the blinded phase of this trial and their allocation is unknown. For these patients, this measure includes prescriptions for "LAl or placebo". For all four patients, "LAl or placebo" accounted for $<8 \%$ of their total amikacin exposure.

All isolates tested resistant to clarithromycin ( $\mathrm{MIC} \geqslant 8 \mu \mathrm{g} \cdot \mathrm{mL}^{-1}$ ), except for the last isolates of two patients $(\mathrm{E}$ and $\mathrm{N})$ (table 4). While initial isolates (E1 and $\mathrm{N} 1$ ) showed inducible clarithromycin resistance (susceptible at 3-5 days but resistant by day 14), the later isolates (E2-1, E2-2, N2-1 and N2-2), tested susceptible to clarithromycin even after 14 days $\left(\right.$ MIC $\leqslant 2 \mu \mathrm{g} \cdot \mathrm{mL}^{-1}$ ) and harboured truncated erm $(41)$ genes. Both first isolates (E1 and N1) were identified as M. abscessus subsp. abscessus and all the last isolates (E2-1, E2-2, N2-1 and N2-2) as M. abscessus subsp. massiliense. Based on the results of genetic sequencing of four genes, erm (41), rpoB, secA and $h s p 65$, E1 was a different strain from isolates E2-1 and E2-2; E2-1 and E2-2 were genetically indistinguishable from each other. For the $\mathrm{N}$ isolates, all three were genetically distinct from each other and were classified as different strains.

Of the 14 remaining patients, first and last isolates from 12 patients showed full-length $\mathrm{erm}(41)$ genes, all with a $\mathrm{T}$ at position 28 and thus expected to confer inducible macrolide resistance. Acquired resistance to clarithromycin was observed in the first and last isolates from patients M and P (A2058C mutation of the $23 \mathrm{SRNA}$ ), which had truncated erm(41) genes. The last isolate from patient K (K2), which had a full-length erm(41), also exhibited acquired resistance to clarithromycin (A2058G mutation of the 23S rRNA), along with acquired aminoglycoside resistance (table 4 and table S1).

\section{Sequence analysis of the erm(41) gene}

By comparison of the erm(41) gene sequences, all of the serial isolates for each patient grouped within their cluster except for patients E and N, whose first isolates E1 and N1 harboured a full erm(41) gene while their last isolates (E2-1, E2-2, N2-1 and N2-2) showed a truncated gene. 13 isolates showed a 100\% match to the erm(41) gene of M. abscessus subsp. abscessus ATCC $19977^{\mathrm{T}}$, corresponding to sequevar 1. Nine

\section{TABLE 3 Other medications prescribed for treatment of Mycobacterium abscessus}

\begin{tabular}{|c|c|c|c|}
\hline Patient & $\begin{array}{l}\text { Duration of amikacin } \\
\text { exposure years }\end{array}$ & $\begin{array}{l}\text { Duration of macrolide } \\
\text { exposure years }\end{array}$ & Other M. abscessus-directed medications \\
\hline A & 8.6 & 10.2 & $\begin{array}{c}\text { Cefoxitin, clofazimine, imipenem, linezolid, meropenem, moxifloxacin, } \\
\text { tedizolid, tigecycline }\end{array}$ \\
\hline B & 4.3 & 8.1 & Linezolid, moxifloxacin, tigecycline \\
\hline C & 1.6 & 9.2 & Linezolid, meropenem, moxifloxacin \\
\hline D & 0.6 & 10.7 & Linezolid, moxifloxacin, tigecycline \\
\hline $\mathbf{E}$ & 2.4 & 3.3 & Imipenem, linezolid, meropenem, moxifloxacin \\
\hline $\mathbf{H}$ & 2.0 & 7.2 & Linezolid \\
\hline I & 2.1 & 7.2 & Clofazimine, linezolid, meropenem, moxifloxacin, tigecycline \\
\hline J & 5.3 & 6.3 & Clofazimine, imipenem, linezolid, meropenem, tigecycline \\
\hline $\mathrm{K}$ & 1.7 & 5.1 & $\begin{array}{c}\text { Bedaquiline, cefoxitin, clofazimine, imipenem, linezolid, meropenem, } \\
\text { tedizolid, tigecycline }\end{array}$ \\
\hline L & 1.1 & 1.6 & Clofazimine, linezolid, meropenem, moxifloxacin \\
\hline
\end{tabular}




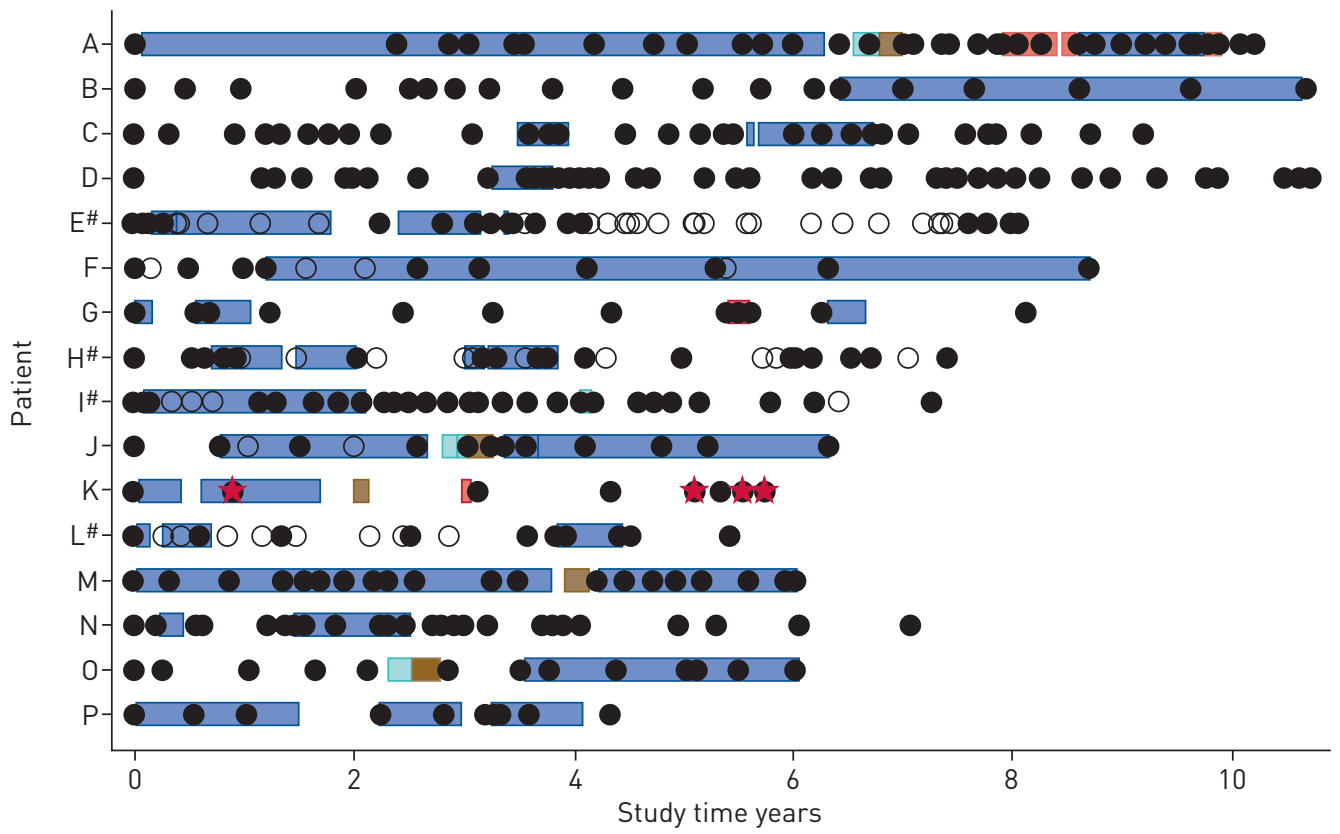

Inhaled amikacin

Liposomal amikacin for inhalation or placebo

Culture positive

$\star$ Amikacin resistance detected

Intravenous amikacin

Liposomal amikacin for inhalation

O Culture negative

\# Culture conversion

FIGURE 1 Timeline of positive and negative patient cultures over the study period relative to amikacin exposure. Amikacin resistance detected: resistance detected either through study assays or through clinical susceptibility testing. Lack of detected resistance for positive cultures is due either to lack of resistance or lack of susceptibility testing.

isolates had a $100 \%$ match to erm(41) gene sequevar 10 [25]. Five strains showed a $100 \%$ match to sequevar 6 and M. abscessus subsp. massiliense CI2040, while O1 showed $99.8 \%$ similarity.

The erm(41) genes of I1 and I2 matched 100\% that of M. abscessus subsp. massiliense isolate CI8182 but were different from published M. abscessus sequevars (only a 98.5\% match to sequevar 6). Isolates CI8182 and CI2040 were included in this manuscript as reference organisms to compare with isolates I1 and I2; CI8182, CI2040, I1 and I2 are all M. abscessus subsp. massiliense with a full erm(41) gene [25]. To the best of our knowledge, this is the first time multiple $M$. abscessus subsp. massiliense isolates with these full-length erm(41) sequevars have been characterised. Partial sequences of the erm(41) gene from strains I1, I2 and O1 were deposited in GenBank under accession numbers MG702334-MG702336, respectively. Eight isolates showed a truncated erm(41) gene (table S1).

\section{Clinical, microbiological and radiographic findings}

Measures of disease severity indicated disease progression in this cohort. Lung function, as measured by forced expiratory volume in $1 \mathrm{~s}$, decreased significantly from a mean \pm SD of $89 \pm 19 \%$ predicted to $69 \pm 22 \%$ predicted, while the number of patients with elevated CRP increased from four of 16 to 11 out of 15 (table 5). Symptoms did not change significantly from the initial to the final visit. The proportion of patients with cavitary lesions increased significantly, from one out of $16(6 \%)$ to seven out of $15(47 \%)$, while the proportion of patients with a Reiff score indicative of severe bronchiectasis did not; most patients had severe bronchiectasis at the time of the first visit (12 out of 16, 75\%) and this severity remained high at the final visit (13 out of 15,87\%). Semiquantitative acid-fast bacillus smear, a measure of bacterial load [9], increased significantly from a median of 1 (few) to 3 (many). Culture quantity also increased, from a median of 3 (scant) to 6 (heavy), although this change was not statistically significant. Four of the sixteen (25\%) study patients experienced culture conversion (figure 1). Thus, although amikacin resistance did not develop in this patient population, as a whole, patients' disease did continue to progress.

\section{Discussion}

In this cohort of patients with $M$. abscessus disease and extended exposure to predominantly inhaled amikacin, only one out of 16 developed amikacin resistance, while on average, disease progressed in this patient group. Past studies have shown the potential for sporadic development of amikacin resistance in 
TABLE 4 Phenotypic and genotypic antibiotic susceptibility results

\begin{tabular}{|c|c|c|c|c|c|c|c|c|}
\hline Isolate & $\begin{array}{l}\text { M. abscessus } \\
\text { subsp. }\end{array}$ & $\begin{array}{l}\text { Date of } \\
\text { isolate }\end{array}$ & $\begin{array}{l}\text { Isolate } \\
\text { type }\end{array}$ & $\begin{array}{l}\text { Clarithromycin MIC at } \\
14 \text { days } \mu \mathrm{g} \cdot \mathrm{mL}^{-1}\end{array}$ & $\begin{array}{l}\text { Constitutive clarithromycin } \\
\text { resistance by } 23 \mathrm{~S} \text { rRNA gene } \\
\text { sequencing }\end{array}$ & $\begin{array}{l}\text { Functional } \\
\text { erm(41) by } \\
\text { sequencing }\end{array}$ & $\begin{array}{l}\text { Amikacin } \\
\text { MIC } \mu \mathrm{g} \cdot \mathrm{mL}^{-1}\end{array}$ & $\begin{array}{c}\text { Amikacin resistance } \\
\text { by } 16 \mathrm{~S} \text { rRNA gene } \\
\text { sequencing }\end{array}$ \\
\hline A2 & abscessus & March 222016 & Rough & $>16(\mathrm{R})$ & No & Yes & 16 (S) & No \\
\hline B1 & abscessus & Oct 122005 & Rough & $>16(R)$ & No & Yes & 16 (S) & No \\
\hline B2 & abscessus & June 102016 & Rough & $>16(\mathrm{R})$ & No & Yes & $32(1)$ & No \\
\hline C1 & abscessus & Feb 62006 & Rough & $>16(R)$ & No & Yes & 16 (S) & No \\
\hline D1-1 & abscessus & Nov 82005 & Rough & $>16(\mathrm{R})$ & No & Yes & 16 (S) & No \\
\hline D1-2 & abscessus & Nov 82005 & Smooth & $>16(R)$ & No & Yes & 32 (I) & No \\
\hline D2 & abscessus & July 132016 & Rough & $>16(R)$ & No & Yes & 32 (I) & No \\
\hline E1 $\#$ & abscessus & Aug 262008 & Smooth & $>16(\mathrm{R})$ & No & Yes & 8 (S) & No \\
\hline E2-1 $\#$ & massiliense & Sept 12016 & Rough & 1 (S) & No & No & $8(S)$ & No \\
\hline E2-2 & massiliense & Sept 12016 & Smooth & 0.12 (S) & No & No & 4 (S) & No \\
\hline F1 & abscessus & Nov 292007 & Rough & $>16(R)$ & No & Yes & $8(S)$ & No \\
\hline H2-1 & abscessus & Dec 12015 & Rough & $>16(\mathrm{R})$ & No & Yes & 16 (S) & No \\
\hline H2-2 & abscessus & Dec 12015 & Smooth & $16(R)$ & No & Yes & 16 (S) & No \\
\hline 11 & massiliense & Aug 52008 & Rough & $>16(R)$ & No & Yes & 8 (s) & No \\
\hline 12 & massiliense & Nov 42015 & Rough & $>16(\mathrm{R})$ & No & Yes & $8(S)$ & No \\
\hline J1 & abscessus & May 112010 & Smooth & $>16(R)$ & No & Yes & $8(S)$ & No \\
\hline J2 & abscessus & Sept 212016 & Smooth & $8(R)$ & No & Yes & 32 (I) & No \\
\hline K1 & abscessus & Sept 212010 & Rough & $>16(R)$ & No & Yes & 16 (S) & No \\
\hline $\mathrm{K} 2$ & abscessus & June 122016 & Rough & $>16(\mathrm{R})$ & Yes & Yes & $>64(\mathrm{R})$ & Yes \\
\hline L1 & abscessus & Jan 42011 & Smooth & $>16(\mathrm{R})$ & No & Yes & $32(1)$ & No \\
\hline L2 & abscessus & June 22016 & Smooth & $>16(\mathrm{R})$ & No & Yes & 16 (S) & No \\
\hline M1 & massiliense & Nov 102009 & Rough & $>16(R)$ & Yes & No & 16 (S) & No \\
\hline M2 & massiliense & Nov 102015 & Rough & $>16(\mathrm{R})$ & Yes & No & 8 (S) & No \\
\hline N1 ${ }^{1}$ & abscessus & April 22009 & Smooth & $>16(\mathrm{R})$ & No & Yes & 4 (S) & No \\
\hline N2-1 1 & massiliense & April 202016 & Rough & $<0.06$ (S) & No & No & 16 (S) & No \\
\hline
\end{tabular}

MIC: minimum inhibitory concentration; rRNA: ribosomal RNA; R: resistant; S: susceptible; I: intermediate. ${ }^{\#}$ : for the E isolates, based on sequencing of four genes, E1 was a different strain from isolates E2-1 and E2-2, which were genetically indistinguishable from each other: १: for the $\mathrm{N}$ isolates, all three were genetically distinct from each other and were classified as different strains.

MAC among patients on inhaled amikacin [18, 27] or LAI [19]. However, rare development of resistance, despite long-term exposure to inhaled amikacin, suggests that this mode of exposure rarely induces resistance in M. abscessus, even at the extremes of treatment duration. Moreover, this study underestimates amikacin exposure, as treatment prior to the study period is not included. Further research could explore hypotheses related to the rarity of amikacin resistance in $M$. abscessus, including insufficient drug penetration or a high barrier to the development of resistance for this species.

Generalisability of these results is limited by selection for length of follow-up with positive sputum culture and thus, effectively, for treatment failure. While the culture conversion rate (for durable culture conversion without relapse) was only $\sim 50 \%$ in a US population of M. abscessus patients similar to those at our centre [4], it was even lower in our study population, with only four (25\%) out of 16 study patients classified as demonstrating culture conversion (with relapse or reinfection) during the study period. However, this rate is similar to other populations of patients with macrolide-resistant M. abscessus [8]. The worsening of disease severity during the study period is therefore not unexpected, given that we have effectively selected for patients who were culture positive at the time of their last visit, and therefore had refractory disease. Other limitations of the generalisability of these results include the almost exclusive use of inhaled amikacin sulfate and the few patients with the fibrocavitary form of the disease, which limit the applicability of the results to patients with $M$. abscessus treated with intravenous amikacin or those with fibrocavitary disease in the context of chronic obstructive pulmonary disease. While progression was not 


\section{TABLE 5 Clinical and radiographic outcomes}

Symptom/severity measure

\section{Clinical}

FEV $1 \%$ predicted mean \pm SD

Elevated C-reactive protein

Cough

Haemoptysis

Fever

Night sweats

Fatigue

Sputum

Shortness of breath

Weight loss

Radiographic

Cavitary

Reiff score $\geqslant 3$ lobes or 1 lobe with cystic BE

Microbiological

Smear quantity*

Negative

Few

Moderate

Many

Culture quantity median (IQR)
Initial visit (n=16)

Final visit $\left(n=15^{\#}\right.$

FEV1: forced expiratory volume in $1 \mathrm{~s}$; $\mathrm{BE}$ : bronchiectasis; IQR: interquartile range. \#: no clinical or radiographic data were available within 365 days of the final visit for one patient, so for these variables, $n=15$; ": according to a semiquantitative scale of 1 (liquid media only), 2 (one colony), 3 (scant), 4 (light), 5 (moderate) and 6 (heavy). ${ }^{*}: p<0.05 ;{ }^{* *}: p<0.01$.

consistent across all radiographic and microbiological measures, this is likely due to the lower variability in Reiff score and smear quantity, and thus lower sensitivity to detect a change in these measures. Moreover, the effective dose of amikacin has not been established [9], and the variable doses used in this cohort may have influenced the development of resistance. Nonetheless, the lack of amikacin resistance in this subset of patients with progressing disease suggests that even in the absence of resistance, amikacin may be insufficient to decrease bacillary burden, although it may slow the rate of disease progression.

In vitro data from a hollow fibre model that mimics amikacin human pulmonary pharmacokinetics provides support for the hypothesis that lower level resistance could result in the observed poor bactericidal activity, leading to continued disease progression $[28,29]$. In this model, despite a decrease in bacterial burden until day 14, bacterial burden increased above initial levels after that point. However, the amikacin-resistant subpopulation did not increase during that time, suggesting that lower level resistance could be leading to the observed poor bactericidal activity. This lack of efficacy is consistent with the described cure rates of $M$. abscessus, which range from $25 \%$ to $48 \%$ among patient populations primarily affected with the commonly macrolide-resistant subspecies M. abscessus subsp. abscessus [4, 8, 30]. Our patient population included patients with both M. abscessus subsp. massiliense and subsp. abscessus, but all initial isolates were resistant to macrolides. These studies highlight the need to prospectively study the efficacy of medication regimens for macrolide-resistant $M$. abscessus and its relationship to the antibiotic susceptibility of patient isolates, particularly to amikacin.

As only one patient in this study developed amikacin resistance ( $\mathrm{MIC}>64 \mu \mathrm{g} \cdot \mathrm{mL}^{-1}$ ), we cannot identify associated risk factors. This patient had cystic fibrosis and had a similar duration of exposure to amikacin compared with other subjects. Of note, the patient was one of three patients to receive intravenous amikacin during the study period; however, this amikacin was administered after the first isolate showing resistance and could not have contributed to its development. Prior to enrolment at the National Institutes of Health, this patient additionally experienced substantial amikacin exposure (including intravenous exposure).

Brown-ElLiotT et al. [13] recently described $10 \mathrm{erm}(41)$ sequevars and the utility of erm sequencing to predict inducible macrolide resistance in $M$. abscessus. As noted previously, plates were evaluated at 3-5 days and 14 days to detect inducible resistance. Strains with MICs $>16 \mu \mathrm{g} \cdot \mathrm{mL}^{-1}$ were associated with seven sequevars (1, 4 and 6-10); the majority belonged to sequevar 1, matching the type strain (ATCC $\left.19977^{\mathrm{T}}\right)$. In our study, all 13 isolates matching sequevar 1 showed clarithromycin MICs in the resistant 
range. Six isolates matching sequevar 6 and nine isolates matching sequevar 10 showed MICs $\geqslant 16 \mu \mathrm{g} \cdot \mathrm{mL}^{-1}$, within the range reported by BROwN-ELLIOTT et al. [13]. Isolates I1 and I2, which harbour a full erm(41) gene sequence matching that of a previously reported M. abscessus subsp. massiliense strain (CI8182) with inducible clarithromycin resistance [25], also showed MICs $\geqslant 16 \mu \mathrm{g} \cdot \mathrm{mL}^{-1}$.

While 14 of the 16 patients presumably had persistent infections with their initial strain, two patients (E and $\mathrm{N}$ ) had first and last isolates from different subspecies. Patient E had a 3-year period of negative cultures, during which treatment was discontinued, suggesting cure and re-infection (figure 1). In contrast, patient $\mathrm{N}$ had consistently positive cultures while on intermittent treatment, indicating the possibility of a mixed infection. Given current culture protocols, the mixed infection may have been present since the first culture, with only the predominant strain being isolated. This finding is consistent with a past study showing $41 \%$ of patients were re-infected with NTM lung disease following successful treatment of $M$. abscessus, including five out of 14 patients who acquired a genetically distinct M. abscessus strain [31].

The limitations of this study include its small sample size and lack of analysis of interim isolates. The sample size was unavoidably limited by resource constraints but the examination of the remaining isolates may have identified further resistance. However, given the selection of patients for significant exposure, we maximised the chance of detecting resistance. Additionally, by analysing only the first and last isolates, we may have missed mixed infections or acquisition of new infections occurring between the first and last isolates. Given the potential for significant genetic diversity of M. abscessus isolates within a patient [32] and that only morphologically distinct colonies were isolated, it is also possible that additional, genetically distinct strains present in the patients were not isolated and thus excluded.

In this cohort of patients with long treatment durations, development of amikacin resistance was rare but outcomes were poor and re-infection occurred in two patients. These findings highlight the need for more effective therapies for macrolide-resistant $M$. abscessus, as well as strategies to prevent re-infection when treatment is successful.

Conflict of interest: None declared.

Support statement: This study was supported by the Intramural Research Programs of the National Institute of Allergy and Infectious Diseases and the National Heart, Lung, and Blood Institute, and the Clinical Center of the National Institutes of Health. Funding information for this article has been deposited with the Crossref Funder Registry.

\section{References}

1 Prevots DR, Marras TK. Epidemiology of human pulmonary infection with nontuberculous mycobacteria: a review. Clin Chest Med 2015; 36: 13-34.

2 Strollo SE, Adjemian J, Adjemian MK, et al. The burden of pulmonary nontuberculous mycobacterial disease in the United States. Ann Am Thorac Soc 2015; 12: 1458-1464.

3 Prevots DR, Shaw PA, Strickland D, et al. Nontuberculous mycobacterial lung disease prevalence at four integrated health care delivery systems. Am J Respir Crit Care Med 2010; 182: 970-976.

4 Jarand J, Levin A, Zhang L, et al. Clinical and microbiologic outcomes in patients receiving treatment for Mycobacterium abscessus pulmonary disease. Clin Infect Dis 2011; 52: 565-571.

5 Cowman S, Burns K, Benson S, et al. The antimicrobial susceptibility of non-tuberculous mycobacteria. J Infect 2016; 72: 324-331.

6 Adekambi T, Sassi M, van Ingen J, et al. Reinstating Mycobacterium massiliense and Mycobacterium bolletii as species of the Mycobacterium abscessus complex. Int J Syst Evol Microbiol 2017; 67: 2726-2730.

7 Griffith DE, Aksamit T, Brown-Elliott BA, et al. An official ATS/IDSA statement: diagnosis, treatment, and prevention of nontuberculous mycobacterial diseases. Am J Respir Crit Care Med 2007; 175: 367-416.

8 Koh WJ, Jeon K, Lee NY, et al. Clinical significance of differentiation of Mycobacterium massiliense from Mycobacterium abscessus. Am J Respir Crit Care Med 2011; 183: 405-410.

9 Olivier KN, Shaw PA, Glaser TS, et al. Inhaled amikacin for treatment of refractory pulmonary nontuberculous mycobacterial disease. Ann Am Thorac Soc 2014; 11: 30-35.

10 Mougari F, Bouziane F, Crockett F, et al. Selection of resistance to clarithromycin in Mycobacterium abscessus subspecies. Antimicrob Agents Chemother 2017; 61: e00943-16.

11 Wallace RJ Jr, Meier A, Brown BA, et al. Genetic basis for clarithromycin resistance among isolates of Mycobacterium chelonae and Mycobacterium abscessus. Antimicrob Agents Chemother 1996; 40: 1676-1681.

12 Bastian S, Veziris N, Roux AL, et al. Assessment of clarithromycin susceptibility in strains belonging to the Mycobacterium abscessus group by erm(41) and rrl sequencing. Antimicrob Agents Chemother 2011; 55: 775-781.

13 Brown-Elliott BA, Vasireddy S, Vasireddy R, et al. Utility of sequencing the erm(41) gene in isolates of Mycobacterium abscessus subsp. abscessus with low and intermediate clarithromycin MICs. J Clin Microbiol 2015, 53: $1211-1215$

14 Kim HY, Kim BJ, Kook Y, et al. Mycobacterium massiliense is differentiated from Mycobacterium abscessus and Mycobacterium bolletii by erythromycin ribosome methyltransferase gene (erm) and clarithromycin susceptibility patterns. Microbiol Immunol 2010; 54: 347-353.

15 Nash KA, Brown-Elliott BA, Wallace RJ Jr. A novel gene, erm(41), confers inducible macrolide resistance to clinical isolates of Mycobacterium abscessus but is absent from Mycobacterium chelonae. Antimicrob Agents Chemother 2009; 53: 1367-1376.

16 Nessar R, Reyrat JM, Murray A, et al. Genetic analysis of new 16S rRNA mutations conferring aminoglycoside resistance in Mycobacterium abscessus. J Antimicrob Chemother 2011; 66: 1719-1724. 
17 Prammananan T, Sander P, Brown BA, et al. A single $16 \mathrm{~S}$ ribosomal RNA substitution is responsible for resistance to amikacin and other 2-deoxystreptamine aminoglycosides in Mycobacterium abscessus and Mycobacterium chelonae. J Infect Dis 1998; 177: 1573-1581.

18 Jhun BW, Yang B, Moon SM, et al. Amikacin inhalation as salvage therapy for refractory nontuberculous mycobacterial lung disease. Antimicrob Agents Chemother 2018; 62: e00011-18.

19 Olivier KN, Griffith DE, Eagle G, et al. Randomized trial of liposomal amikacin for inhalation in nontuberculous mycobacterial lung disease. Am J Respir Crit Care Med 2017; 195: 814-823.

20 Kim RD, Greenberg DE, Ehrmantraut ME, et al. Pulmonary nontuberculous mycobacterial disease: prospective study of a distinct preexisting syndrome. Am J Respir Crit Care Med 2008; 178: 1066-1074.

21 Chalmers JD, Goeminne P, Aliberti S, et al. The bronchiectasis severity index. An international derivation and validation study. Am J Respir Crit Care Med 2014; 189: 576-585.

22 van Ingen J, Aksamit T, Andrejak $\mathrm{C}$, et al. Treatment outcome definitions in nontuberculous mycobacterial pulmonary disease: an NTM-NET consensus statement. Eur Respir J 2018; 51: 1800170.

23 Clinical and Laboratory Standards Institute. Susceptibility testing of mycobacteria, nocardiae, and other aerobic actinomycetes; approved standard. 2nd Edn. Wayne, CLSI, 2011.

24 Shallom SJ, Moura NS, Olivier KN, et al. New real-time PCR assays for detection of inducible and acquired clarithromycin resistance in the Mycobacterium abscessus group. J Clin Microbiol 2015; 53: 3430-3437.

25 Shallom SJ, Gardina PJ, Myers TG, et al. New rapid scheme for distinguishing the subspecies of the Mycobacterium abscessus group and identifying Mycobacterium massiliense isolates with inducible clarithromycin resistance. J Clin Microbiol 2013; 51: 2943-2949.

26 Zelazny AM, Root JM, Shea YR, et al. Cohort study of molecular identification and typing of Mycobacterium abscessus, Mycobacterium massiliense, and Mycobacterium bolletii. J Clin Microbiol 2009; 47: 1985-1995.

27 Brown-Elliott BA, Iakhiaeva E, Griffith DE, et al. In vitro activity of amikacin against isolates of Mycobacterium avium complex with proposed MIC breakpoints and finding of a 16S rRNA gene mutation in treated isolates. J Clin Microbiol 2013; 51: 3389-3394.

28 Ferro BE, Srivastava S, Deshpande D, et al. Failure of the amikacin, cefoxitin, and clarithromycin combination regimen for treating pulmonary Mycobacterium abscessus infection. Antimicrob Agents Chemother 2016; 60: 6374-6376.

29 Ferro BE, Srivastava S, Deshpande D, et al. Amikacin pharmacokinetics/pharmacodynamics in a novel hollow-fiber Mycobacterium abscessus disease model. Antimicrob Agents Chemother 2015; 60: 1242-1248.

30 Harada T, Akiyama Y, Kurashima A, et al. Clinical and microbiological differences between Mycobacterium abscessus and Mycobacterium massiliense lung diseases. J Clin Microbiol 2012; 50: 3556-3561.

31 Koh WJ, Jeong BH, Kim SY, et al. Mycobacterial characteristics and treatment outcomes in Mycobacterium abscessus lung disease. Clin Infect Dis 2017; 64: 309-316.

32 Bryant JM, Grogono DM, Rodriguez-Rincon D, et al. Emergence and spread of a human-transmissible multidrug-resistant nontuberculous mycobacterium. Science 2016; 354: 751-757. 